\title{
Design of Digital Circuit Online Examination System
}

\author{
Qian Cheng, Ziying Wang, and Xiaodan Wang
}

\begin{abstract}
In view of the limitations of the existing web-based examination system, a "digital circuit" online examination system was designed. This system is mainly made up of rich graphical interface in front-end development technology such as HTML5, and the asynchronous update function of Ajax technology. It not only realized online examination of special questions such as timing diagram type, karnaugh map grouping and circuit design, but also improves the operation efficiency and storage security of $\mathrm{B} / \mathrm{S}$ architecture examination system.
\end{abstract}

Index Terms-Digital circuit, B/S architecture, HTML 5, Ajax.

\section{INTRODUCTION}

Examination is an important link in the teaching process, and the examinations of special subjects are limited by complex expressions. It is always difficult to test students' comprehensive application ability. People have always relied on the paper exam, but traditional exams are often unsafe, costly and unfair [1], this method often makes people subject to various restrictions such as time and space, causing a lot of inconvenience.

Nowadays, with the rapid development of IT technology and Internet, examination network is the development trend in the future, and the existing examination methods, such as examination system with $\mathrm{C} / \mathrm{S}$ architecture, also exist. This test system relies on software, but there are still many problems in the actual operation. For example, there might be a problem in the software which leads to the inability to test and cause great trouble [2]. But online exam can avoid many problems. It's more convenient to create test paper, answer the paper, and mark it. It not only solves a lot of tedious work problems in traditional exams, but also creates favorable conditions for students' learning and teachers' teaching management to realize the real "paperless" exam. General networked test in the design of special topic often only supports the manual group volume, This method is often time consuming and laborious, and the quality of examination papers is often different from person to person, which is also difficult to be controlled. Therefore, the system often chooses to fill in these side questions to investigate students. In this way, it is often impossible to truly examine students' practical ability in specific subjects.

In order to solve this problem, this paper uses web related front-end and database technology to design and develop an online examination system to support web page answer, and it can realize the construction of special questions.

Manuscript received October 11, 2018; revised May 1, 2019.

The authors are with the College of engineer, Yanbian University, Jilin, China (e-mail: wangxiaodan@ybu.edu.cn).

\section{DeVelopment TeChNOlOgY AdOPTED By THE SySTEM}

This system adopts PHP language, based on Windows platform, Mysq15.7 database development, and adopts Bootstrap, Ajax technology, HTML, CSS and other technologies at the browser end. The server side adopts Struts2 and Hibernate4 framework which implement the MVC pattern. The following is a brief introduction of the development technology used in the system.

PHP language is powerful, easy to learn and easy to use. And it's a common open source scripting language. Whose syntax absorbs the features of C language, Java and Perl, is good for learning and wide use, and is mainly applicable to areas such as Web development. Whose unique syntax blends C, Java, Perl, and its own syntax. It can execute dynamic web pages more quickly than CGI or Perl.

MVC organizes code using a separate method of business logic, data, and interface display, aggregating business logic into a component that improves and customizes the interface and user interaction without having to rewrite the business logic[3].

Bootstrap is a front-end rapid development framework based on CSS, HTML and JavaScript, which makes Web development faster. Bootstrap provides elegant HTML and CSS specifications written in dynamic CSS language Less.

Ajax technology is primarily a partial update technology that can update parts of a web page without reloading the entire web page. By exchanging a small amount of data with the server in the background [4], Ajax enables pages to be updated asynchronously. This means that parts of the page can be updated without reloading the entire page.

The system is mainly developed with XAMPP, which integrates Apache+MySQL+ PHP+PERL. Its biggest advantage is that it is extremely convenient, easy to install and to use to achieve powerful functions.

\section{SySTEM DESIGN AND IMPLEMENTATION}

\section{A. Overall Structure}

The functions of the special online examination system are as follows. Firstly, the system can identify the identity of the user. Second, examinees can log into the test system on any internet-connected computer with a browser, complete the test paper and submit it within the specified time. Third, at the end of the examination, the examination system can achieve a sound and rigorous evaluation function; Fourthly, the teacher can conveniently manage the related information of the examination, add, delete and revise the examination database; Fifth, background administrators can modify the information of students and teachers. They can maintain and update the system regularly. 
According to the above functional requirements, this paper designs the whole system from the perspective of detection and management

Students entrance of exam-ination is login interface. And the verification makes use of MysqlConnect to call for the background database automatically. Each time the tester enter the online exam, relevant information of the examinee is displayed in the upper left corner, the remaining time of the exam is automatically displayed in the upper right of the system, and various questions are displayed in the middle, then the tester click and go to the question. The next section details the design of the test system.

The teacher system mainly provides the administrators and teachers with the functions of artificial grouping, intelligent grouping, question bank management, question management, examination paper management and performance management. When entering the system, the upper left corner will indicate the login information of the teacher, and system displays the list of questions, examination papers, and performance management. The teacher can browse the questions by himself for selection, and then select and select the questions to for testing. When clicking on performance management, teacher's performance management system will first sentenced to roll into the interface, the upper left shows the topic type of ID, the examinee answer by the data, and the examinee answer timing diagram question is converted into an array. The teacher only needs to compare with the standard answer or set up the system to automatically compare to get the result quickly, and no longer need to repeatedly browse the information irrelevant to the answer, which will greatly improve the efficiency of marking. In addition, the most important thing of the teacher side is to ensure that every operation of the user is verified, and that the user cannot use the function provided by the system when the user is not logged in or directly enters the address.

This paper designed the communication protocol between the front-end and back-office server.

Of course, the most important front-end of the system includes various question type design, such as multiple-choice question type, fill-in-the-blank, judgment, short-answer question, as well as the most characteristic design question and timing diagram question design, which will be discussed in detail in the following part.

\section{B. Background Database Design System}

The whole system adopts the MVC design pattern to plan the whole background operation, and uses the method of separation of business logic, data and interface display to organize the code to make the whole system more perfect and reliable. The main tables in the database include the student user login information table, teacher user table, question design table, etc., which are used to store the data related to the test system. The system will separately set up a login interface to realize MySQL's operation of calling the database, and the PHP statement will be invoked every time the login is verified, for analysis and comparison in the database. Similarly, every time the exam is finished, the candidates are sent to the database to be stored in the database, and when the teacher rolls, it's called again.

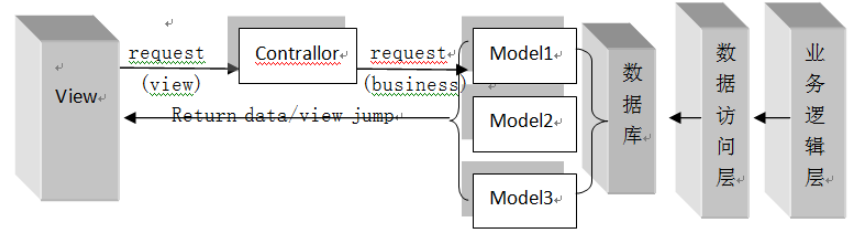

Fig. 1. Database schema design.

\section{Design and Realization of Subjective Question}

Select, fill in the blank, judge and answer questions are all necessary questions in the general test system, which are used to examine some knowledge points that are not easy to directly express the narration from an objective perspective. On this basis, this online system is characterized by the realization of subjective examination, so the counter design questions and timing diagram questions will be introduced in detail.

\section{Counter Design Question}

There are six steps in the counter design question: state diagram, next-state table, flip-flop transition table, Karnuaph maps, logic expressions and counter implementation. Users need to check the contents of other steps again and again. So the accordion effect spreads the powerful superiority to enhance the relevance between the steps, reduce the answer page, facilitate the examinee to compare any steps to answer questions.

The first step of counter design is the state diagram. The system is beautified with standard CSS, and the overall layout uses div layer to divide the page effectively and reasonably. To examinee's each kind of demand, JavaScript is used concretely to increase its interactivity. When the user enters a new status change in the preselected box and clicks the Add button, the data is immediately sent to the form, and the page will trigger the event. The status graph generates a new circle section that jumps to a new image for counting and writes the form sent data in the new circle. Through repeated operations, candidates can achieve a time series of state display.

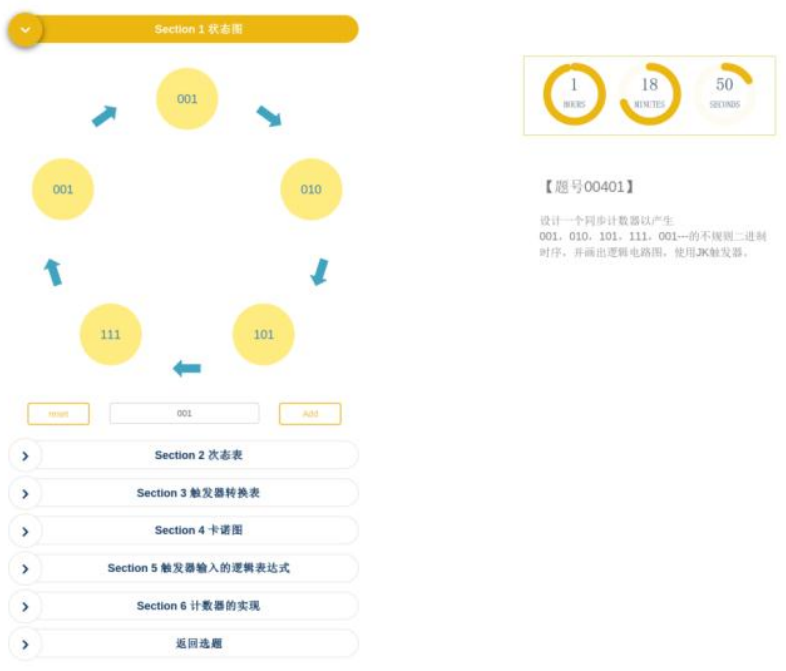

Fig. 2. time series state.

In the second and third steps of the next-state table, flip-flop transition table, the system uses tables to process the data filled in by examinees, and sends the data to the backstage storage, then updates the examinee response 
information table stored in the database in a timely manner. The Collapse effect based on Bootstrap framework is reflected throughout the process, which is currently commonly used accordion effect. This effect is characterized by the previous layout structure. The system uses the plugin to make it easy to fold up the page area and further optimize the interface by clicking on the title to display or hide the corresponding content. And the most critical part of the accordion effect is that each title should have one content. In the Bootstrap framework, these two parts are combined together to be called a panel page. When these panels are combined together, it is a panel combination panel-group, namely the accordion structure.

To tie the title and content areas together, the system used the anchor link method to connect the title area to the panel area to achieve some unique effects. In the Bootstrap framework, the local area is not visible when the candidate fails to answer, and the system will realize this function by adding the collapse on the panel-collapse style. The system can timely activate the accordion interaction in the interaction with the examinee, so the system can customize two attributes in the title link: one is data-toggle, whose value is collapse; The other is data-target, which is the identifier of each panel content area. And the system defines the data-parent attribute. When the candidate implements clicking on one of the elements, the system closes all the collapse areas and opens the clicked areas (if they are shown). This data-parent value matches the identifier of the accordion panel container, So in the fourth step of the carnot diagram, for example to implement the transformation of $0,1, x$, the system uses the above points. The table is hidden at the beginning, so that candidates can click the mouse to realize the number of switching at the same time. After the examinee determines the number in the table, the examinee can click the circle to realize the connection with the mouse. The system mainly adopts the sliding library of HTML effect. After determining a circle, the examinee can drag the mouse to the adjacent box to realize the effect of the circle together.
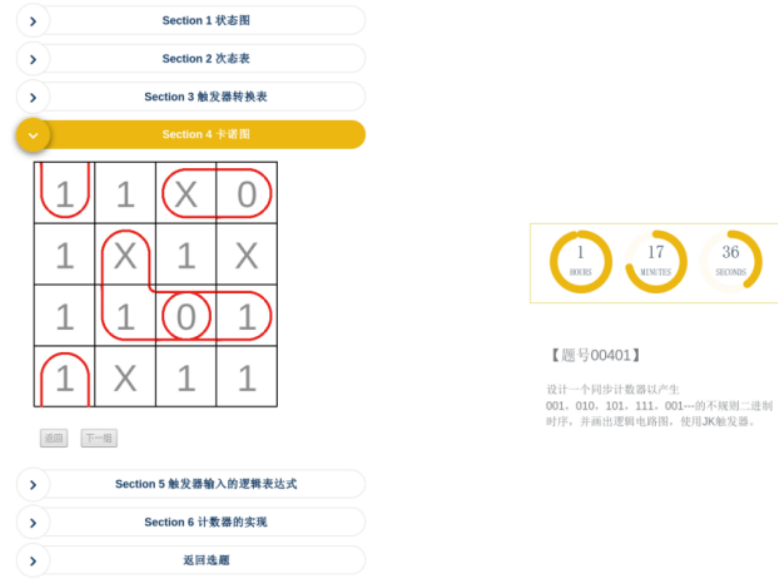

Fig. 3. example karnaugh diagram.

In the fifth step of the counter design question, the system sets up a button for the examinee considering the tedious subscript input. When the examinee clicks, the trigger event will output the corresponding subscript in the box. In the sixth step, considering the diversity of the answers to the design questions of each counter, the system adopts drawing board to connect the counters. In this way, after the end of the test, every picture completed by the examinee is saved to the background database, Of course, the system added the function of clearing the drawing board to clear the trace of drawing when the examinee made a mistake, added the function of exporting the drawing board, and used the relevant function after examinee's answer, turning the canvas into an image for subsequent operations. At last, all the data of the counter questions are saved. The answer data of all the previous steps will be sent to the background database, and will be collected into the answer information table of examinees for use in batch volume.

\section{E. Timing Diagram Inscribes}

The system mainly designs button sets for the waveform to be changed and clicks events for pressing the level button, The event trigger is constructed using a function of a new conversion level, The main logical structure of the function body is to judge the level of the previous button and the level of the latter button every time a level conversion is made, and then the system changes it, This ensures that each level change is correct. Finally, the candidate's submission button system also uses the button that is bound to the click event. Then, the system converts the clicked high and low level into an array and sends it to the final console and background database for storage, so that the final data can be used by readers more concisely.

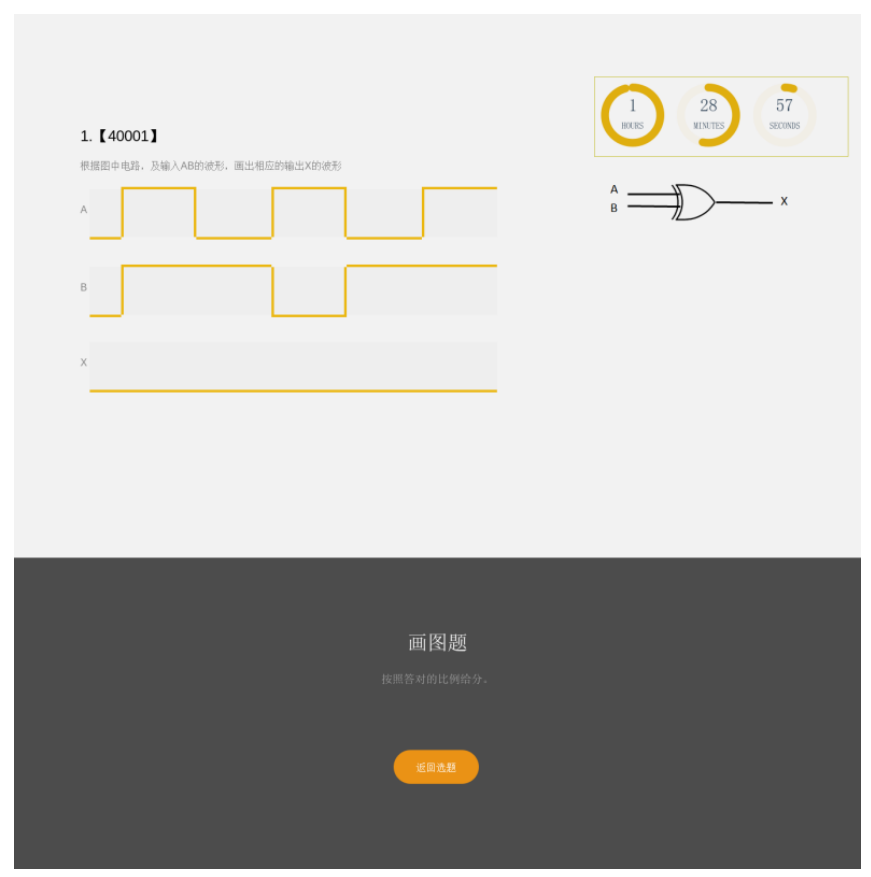

Fig. 4. timing diagram example.

\section{F. Submission of System Data}

This system is an online examination system based on AJAX technology, which can provide a smoother operation interface for administrators and examinees, making it more convenient for administrators to manage the examination database and examinee information. When examinee carries on the online examination, the examination is more fluent, examinee selects the question, answers the question faster, has saved the network broadband greatly, reduces the server 
burden. The submission of system data also uses AJAX technology. By using its unique technology, the asynchronous effect of simultaneous submission can be achieved when examinees answer questions. When the examinee completes a question type answer every time and returns to choose the topic homepage, system can transmit asynchronously to the server through the browser in real time, and passes into examinee database after processing by the server side at the same time. The final operation of the examinee communicates directly with the backstage database, so as to directly avoid the system paralysis caused by the final swarm. Therefore, this partial update operation significantly solves a lot of problems related to the server, and more importantly, it can safely and effectively save the students' answers.

In addition, since the use environment of the $\mathrm{B} / \mathrm{S}$ mode system is relatively complex at present, which is mainly reflected in the large number of prevailing browsers at present and multiple versions of the same browser, the system has been tested for compatibility. For compatibility, 360, Google, Firefox and other browsers have been tested, and the system can be used normally on the browser under test.

\section{CONCLUSIONS AND PROSPECTS}

Based on the web-based examination system, this paper makes use of HTML5 and other related technologies and the asynchronous update function of Ajax technology to realize the "web-based" digital circuit examination system and realize online inspection of special questions such as timing diagram questions and counter design questions. Through systematic testing and trial operation, the system can accurately meet the requirements of the system, and the digital circuit online examination can be conducted stably.Through the course examination, the system fully mobilize the enthusiasm, initiative and consciousness of students in learning the course, and guide them to learn the comprehensive development of students' knowledge, ability and accomplishment to meet the needs and development of the society. [5]

However, in the actual operation process, the system's shortcomings are also exposed. For example, the system management function is not rich enough, and more diversified functions are needed to make the system more complete. In the later stage, more background frameworks will be used to enrich the statistical functions of the test system.

\section{REFERENCES}

[1] J. H. Shuang, "A brief analyses of the development status and trend of online examination system Xi an traffic engineering college,xi'an," 2018.

[2] Y. X. Zhao, "Design and implementation of online examination system," Guangzhou: South China University of Technology, 2015.

[3] Y. X. Zhao and Z. Y. Teng, "JMVC model of J2EE distributed application system based on MVC design pattern," Computer Application Research, 2003, vol. 20, no. 18, pp. 63 64.

[4] G. Ping, "Huang Improving online examination system with Ajax technology," Journal of Nantong Vocational University, 2006, vol. 20, no. 3, pp. 87-89.

[5] L. H. Liu "Exploration and practice of the examination mode of "digital circuit" course in higher vocational colleges," Beijing Institute of Technology, Beijing 100042, China.

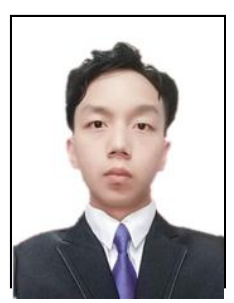

Qian Cheng was born in Wuhan city, HuBei province. he is an undergraduate at Yanbian University, majoring in computer science and technology.

He participated in many technology competitions and won awards, he also won the scholarships for outstanding students many times .He excelled at school, often developed projects with his mentor, and had a strong interest in science.

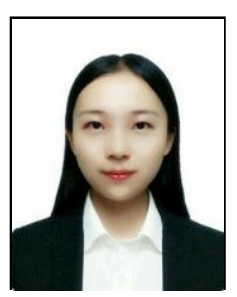

Ziying Wang was born in Rizhao city, Shan dong province. She is an undergraduate at Yanbian University, majoring in computer science and technology.

She was awarded the national scholarship and won the first class scholarship many times during her school year. She has excellent performance and good scientific research ability.

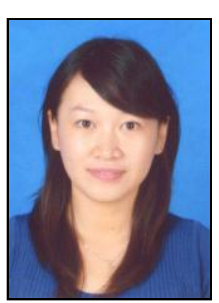

Xiaodan Wang was born in Yanji City, Ji Lin province, in 1981. She has a master's degree in computer application technology and a bachelor's degree in electrical \& information engineering at Yanbian University.

At present, she is committed to interdisciplinary research, such as the integration of computers and machinery or electronics. And she has obtained the certificate of Senior Software Engineer and senior web designer. This year, the students she guided won the first prize in the computer design competition of Jilin district. 